\title{
Peri-transplant renal dysfunction in patients with non-alcoholic steatohepatitis undergoing liver transplantation
}

\author{
Rakhi Maiwall ${ }^{1}$, Manasvi Gupta ${ }^{2}$ \\ ${ }^{1}$ Department of Hepatology, Institute of Liver and Biliary Sciences, New Delhi, India; ${ }^{2}$ Department of Internal Medicine, University of Connecticut \\ School of Medicine, Hartford, CT, USA \\ Contributions: (I) Conception and design: None; (II) Administrative support: None; (III) Provision of study materials or patients: None; (IV) \\ Collection and assembly of data: None; (V) Data analysis and interpretation: None; (VI) Manuscript writing: All authors; (VII) Final approval of \\ manuscript: All authors. \\ Correspondence to: Dr. Rakhi Maiwall, MD, DM. Additional Professor, Department of Hepatology, Institute of Liver \& Biliary Sciences (ILBS), D-1, \\ Vasant Kunj, New Delhi 110 070, India. Email: rakhi_2011@yahoo.co.in; drrakhimaiwall@gmail.com.
}

\begin{abstract}
Non-alcoholic fatty liver disease (NAFLD) is currently the most common etiology of chronic liver disease (CLD) caused by an accumulation of fat in the liver and globally is the leading indication of liver transplantation. Emerging data has recognized an increased association of NAFLD with risk of other metabolic liver diseases like type 2 diabetes mellitus, chronic kidney disease (CKD) and cardiovascular diseases. Pathophysiologically, NAFLD patients have a state of low-grade systemic inflammation, insulin resistance and atherogenic dyslipidemia which causes renal dysfunction. Patients with NAFLD cirrhosis awaiting liver transplant (LT) face unique challenges and have a significantly higher requirement of simultaneous-liver-kidney transplant as compared to other etiologies. Further, NAFLD not only recurs but also occurs as a de novo manifestation post-LT. There is also a significantly higher risk of waiting list stagnation and dropouts due to burdensome cardiometabolic disorders in NAFLD patients. The current review aims to understand the prevalence and pathogenetic basis of renal dysfunction in NAFLD. Additionally, the review describes the choice of immunosuppression protocols and use of intraoperative renal replacement therapy in context of intra and post-operative renal dysfunction in NAFLD patients. Prospective controlled trials focusing on NAFLD and development of CKD are needed to assess the existence of a causal and/or a bidirectional relationship between NAFLD and CKD.
\end{abstract}

Keywords: Non-alcoholic fatty liver disease (NAFLD); cardio-metabolic disorders; hypertension; diabetes; dyslipidemia; chronic kidney disease (CKD); obesity; gut dysbiosis

Received: 23 July 2019; Accepted: 15 October 2019; Published: 05 April 2020.

doi: $10.21037 /$ tgh.2019.10.11

View this article at: http://dx.doi.org/10.21037/tgh.2019.10.11

\section{Introduction}

Nonalcoholic steatohepatitis (NASH) has been on a rising trend with current statistics showing a prevalence of $20-30 \%$ in adults in the Western population (1), while it is $16.9 \%$ in the Asian population $(2,3)$. The spectrum of disease starts from fatty liver and often progresses to NASH. Affected liver, if timely interventions are not made, will eventually progress to cirrhosis and may also present with its complications such as decompensation and hepatocellular carcinoma $(4,5)$. This fast rising cause of chronic liver disease (CLD) is now also emerging as a risk factor for chronic kidney disease (CKD) with evidence favouring the association between kidney disease and NASH, independent of other confounders such as hypertension, diabetes and obesity (6). Since NASH often exists in a syndromic form with other metabolic abnormalities, it becomes important to examine the comorbidities seen in this population and the concurrent effect these have on renal function, before and after transplant. In recent years, as per United Network 
for Organ Sharing (UNOS) data, NASH is currently the second most common cause of liver transplant (LT) in the United States and controversial indication for simultaneous liver kidney transplantation (SLKT). CKD is an important determinant of outcome and long-term mortality and morbidity in post-LT patients (7) The focus of this paper is to examine the renal function in patients of CLD with etiology as NASH before and after LT. This paper further examines possible modifications that can be made in the pre, intraoperative and post-LT care (immunosuppressive regimens, follow-up and renal function monitoring) to avoid long-term renal complications in these patients.

\section{Association of NAFLD with CKD}

A strong association between NAFLD and CKD has been demonstrated by cross-sectional and cohort studies and has been consistent across different patient populations (8). Pacifico and colleagues showed renal functional alterations in children with NAFLD. In a cohort of 596 obese children, they compared 268 children diagnosed with NAFLD on magnetic resonance imaging with 328 children without NAFLD, and 130 healthy controls who had normal weight. They found a greater prevalence of renal dysfunction based on estimated GFR below $90 \mathrm{~mL} / \mathrm{min} / \mathrm{m}^{2}$ in children with NAFLD compared to other groups. Further, the proportion of children with abnormal urine microscopy and evidence of higher albumin excretion was noted in the NAFLD group when compared to other groups without NAFLD after controlling for other relevant clinical and biochemical factors (9). There have been two meta-analysis exploring the association of NAFLD with the development of incident CKD. In the initial systemic review and meta-analysis, Musso and colleagues (10) evaluated whether the presence and severity of NAFLD influence the development and degree of renal dysfunction. They included observational studies assessing NAFLD and studies defining CKD based on either estimated glomerular filtration rate (eGFR) $<60 \mathrm{~mL} / \mathrm{min} / 1.73 \mathrm{~m}^{2}$ or presence of proteinuria. Data from 33 studies (cross-sectional and cohort) including 63,902 patients were analysed. The results of this meta-analysis demonstrated NAFLD as a risk factor for development of incident CKD. They found that the severity of NAFLD directly correlated with an increased risk of CKD as patients with fibrosis had the highest risk of CKD as compared to patients without significant fibrosis. Additionally, patients with steatohepatitis had a higher prevalence of CKD as compared to those with simple steatosis.
In the most recent meta-analysis by Mantovani et al. who did a random-effect modelling using 9 prospective observational cohorts including 96,595 participants (34.1\% with NAFLD and 4,653 cases of incident CKD (defined as occurrence of eGFR $<60 \mathrm{~mL} / \mathrm{min} / 1.73 \mathrm{~m}^{2}$, with or without accompanying overt proteinuria) followed over a median period of 5.2 years. Similar to the results by Musso and colleagues, they observed a direct association of severity of NAFLD with the development of CKD (11). Overall, the data derived from these studies clearly suggest a strong association between NAFLD and CKD.

\section{Metabolic risk factors in NASH associated renal dysfunction}

\section{Obesity}

Obesity and its related diseases which were traditionally considered as an epidemic in the Western countries have now made a global impact. In the last two decades. an increase in the burden of obesity has also been reported from the Asian countries (12). Both the presence and severity of obesity correlates with severity of NAFLD. Almost, two-thirds of NAFLD patients are obese (13). Obesity, especially central type, is associated with physical inactivity, chronic systemic inflammation and insulin resistance which together contribute to renal dysfunction. Progression of CKD in obese is independent of the underlying status of the kidneys. The glomerulopathy in obesity has been coined as obesity related glomerulopathy (ORG) which is associated with focal segmental glomerulosclerosis. Recent data has demonstrated the pitfalls of ectopic fat distribution in obese which is associated with architectural distortion, functional alterations of the mesangial cells, podocytes and the proximal tubular epithelial cells causing renal dysfunction. This is secondary to a maladaptive response to hyperfiltration and albuminuria (14). Salvatore et al. aimed to identify the spectrum of renal changes in obese patients with body mass index (BMI) of $>30 \mathrm{~kg} / \mathrm{m}^{2}$ with CKD. They analysed retrospectively the kidney biopsies of patients and measured the glomerular diameter with a standard micrometer. They found the highest level of proteinuria in patients with idiopathic focal segmental glomerulosclerosis (FSGS) and found that obese-related FSGS mainly had subnephrotic proteinuria. Moreover, the creatinine levels were highest in tubulointerstitial diseases. The results of the study suggested a diverse spectrum of renal abnormalities with a background ORG in patients with obesity (15). 
Recent studies have identified obesity as an independent predictor of progression of the underlying kidney disease. In a longitudinal cohort study, BMI was identified as an independent risk factor for CKD after adjusting for the known risk factors i.e., patient demographics and status of insulin resistance, presence of dyslipidemia and hyperglycemia (16). The risk was highest for patients with class 3 obesity. Similar to this, in the HUNT-1 study an independent risk of end-stage renal disease was noted for patients with class 2 obesity or higher (17). This is because insulin resistance which is so frequent in patients with NASH has been identified as a key mechanism behind the development of renal dysfunction in the obese (18).

Obesity also impacts the renal function by causing hyperfiltration, hyperperfusion and focal glomerulosclerosis with impairment of both the glomerular and tubular function $(19,20)$. Tomaszewski and colleagues in a crosssectional study evaluated 1,572 healthy men and discovered that patients who had glomerular hyperfiltration were not only more frequently obese but also had higher prevalence of cardiometabolic risk factors. Similar to this, in the other epidemiological studies, glomerular hyperfiltration has been demonstrated as a precursor to development of CKD in obese individuals with insulin resistance (21). Biopsies have clearly demonstrated that histological changes precede functional decline in kidney functions in obese patients (22). Therefore, novel clinical biomarkers to identify renal fat using the lipidomic approaches in plasma or urine, adipophilin staining on biopsies or metabolic imaging could be exciting approaches to identify renal damage allowing interventions to prevent progression to CKD in the obese (22). This is because weight reduction has been associated with not only normalization of renal functions in a meta-analysis with reversal of albuminuria but also prevented further decline in renal functions. This highlights the significance of reversibility when interventions are made early in kidney disease in obese patients with metabolic syndrome (23).

\section{Dyslipidemia}

Dyslipidemia with raised serum cholesterol levels is frequently encountered in patients with NAFLD. Serum cholesterol levels serve as a toxic trigger and have been associated with increased progression to cirrhosis by a mechanism mediated through depletion of mitochondrial glutathione which results in hepatocyte necrosis. In a seminal study by John Moorhead, a clear association of hyperlipidemia with renal disease was postulated. Development of significant renal disease is heralded by the deposition of renal fat causing glomerulosclerosis. Its known that the adipose tissue generates high concentrations of circulating non-esterified fatty acids (NEFA). These are subsequently carried to the liver wherein positive signal follows for the hepatic synthesis of very-low density lipoproteins (VLDL) (24). Concomitantly, there is an increased delivery of triglycerides to various nonadipose peripheral tissues. The lipoprotein lipase-mediates subsequent re-esterification of triglycerides which are stored as small low-density lipoproteins (25). The deposition of lipids in the non-adipose tissues i.e., in the liver, heart, muscle and kidneys is a surrogate marker of metabolically unhealthy state in an individual. A bidirectional relationship between ectopic liver fat as in NAFLD and CKD has been recently reported. It has been shown that ectopic liver fat drives development of CKD (26) and CKD might itself cause lipid redistribution from the adipose tissue to nonadipose ectopic locations such as the liver causing NAFLD. Similar to fatty liver, the infiltration of fat in the kidney has now been called as fatty kidney or renal steatosis in patients with obesity or metabolic syndrome (27). The exact amount of lipid in the kidneys which leads to renal dysfunction however is still not known. In the kidneys fat accumulation has been demonstrated in the glomerular structures i.e., the mesangium or the podocytes causing structural and functional alterations as well as the proximal tubule cells which together drive the development of nephropathy in the affected individuals. Almost, $45 \%$ diminished number of podocytes have been reported in the glomerulopathy associated with metabolic syndrome (28). Among patients with stage $\mathrm{V}$ CKD on hemodialysis a significant prevalence of cardiovascular or atherogenic dyslipidemia has been reported. It has further been shown that statins by reducing LDL decrease the risk of cardiovascular events in patients with CKD not requiring dialysis but at the same time these benefits are not encountered in patients on dialysis (29). In a large prospective cohort study of 3,939 patients, none of the lipids or lipoprotein components were independently associated with progression of kidney disease (30).

\section{Diabetes mellitus}

Both NAFLD and type 2 DM share a complex and intricate link with multiple common pro-inflammatory, prothrombotic and proatherogenic pathways leading to fibrosis and several extensive studies have indicated that more than $70 \%$ of patients with type 2 DM develop NAFLD (31-34). Vice-versa to this, two large meta- 


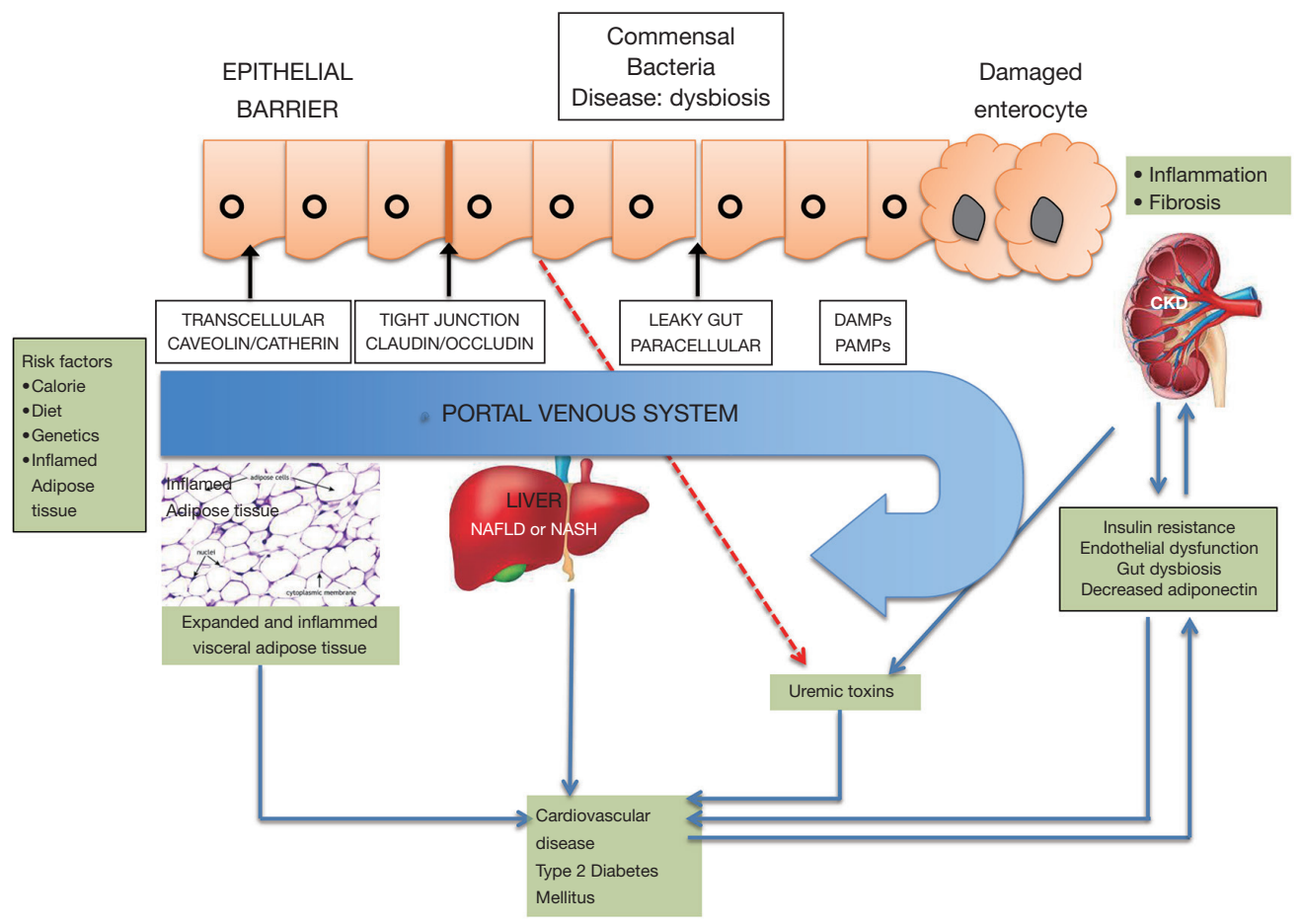

Figure 1 Organ crosstalk in the pathophysiology of NAFLD and CKD. Multiple environmental and genetic factors adipose tissue inflammation can predispose to NAFLD. NEFAs from the intra-abdominal visceral adipose tissue cause activation of inflammation by increasing the levels of NF- $\mathrm{KB}$ and inflammatory pathways, dysregulation of adipokine production and impairment of insulin signaling. With increasing severity or progression of disease there is an increase in the pathways that influence the development of CKD. Increased production of uraemic toxins is seen by concomitant gut dysbiosis with an increase in the intestinal permeability. Intestinal microbiota further leads to renal, liver and cardiovascular damage via endothelial dysfunction in patients with NAFLD and CKD. Intestinal dysbiosis frequently occurs in obesity, potentially influences NAFLD, CKD, and T2DM through complex mechanisms (8). DAMPS-Damage associated molecular patterns, PAMPS-pathogen associated molecular patterns; ROS, reactive oxygen species; CKD, chronic kidney disease; NAFLD, nonalcoholic fatty liver disease; T2DM, type 2 diabetes mellitus.

analysis and several cross-sectional studies have showed that NAFLD patients are predisposed to develop diabetes (35-39). Presence of diabetes has also been shown to correlate with worsening of fibrosis in patients with NAFLD (40-43). Targher and colleagues in a prospective cohort study followed 1,760 participants for almost 6.5 years with type 2 diabetes without CKD. In their study a total of 547 (31\%) patients developed CKD. A significant association of NAFLD with development of CKD was observed after adjusting for other relevant cardiometabolic factors including patient demographics (44). The same investigators also assessed whether NAFLD is associated with development of CKD in patients with type 1 diabetes. They followed a total of 261 type 1 diabetic adults without CKD for a mean period of 5.2 years for the development of CKD. NAFLD was diagnosed based on ultrasonography in this study was shown to be an independent risk factor for incident CKD (45). In another recent study of patients with NAFLD, impaired renal functions were observed in almost a third of patients. Further, the prevalence of renal impairment was related to the severity of liver disease as well as presence of diabetes mellitus (46).

\section{Pathophysiologic basis for renal dysfunction in NASH patients}

Multiple studies have been done in the last 2 years exploring the complex relationship between NASH and renal disease after adjusting for confounders such as diabetes, hypertension and obesity (Figure 1). Even though the metabolic abnormalities coexisting in this patient cohort also adversely affect the renal function, NASH is 
an independent driver of renal dysfunction with multiple hypothesized albeit unproven mechanisms of pathogenesis. Vice-versa, there may also be possible pathogenic pathways that may start with a malfunctioning kidney and eventually cause hepatocyte injury to result in NAFLD (8).

A state of low-grade inflammation has been noted in NAFLD as well as the cardiometabolic phenotypes commonly associated with the disease i.e., T2DM and cardiovascular disease. A plethora of factors are instrumental in the liver lipid accumulation which characterizes NAFLD; and factors like insulin resistance and endothelial cell activation become instrumental in the subsequent disease course which influences the function of extrahepatic organs.

\section{Environmental factors}

Consumption of excessive calories or increased dietary intake of fructose results in increase in de-novo hepatic lipogenesis as well as causes hyperuricemia (47). Increase in uric acid with a concomitant increase in urinary excretion of uric acid could cause renal dysfunction in NAFLD patients (47).

\section{Insulin resistance and systemic inflammation}

In patients with NASH, the inflamed visceral adipose tissue not only promotes insulin resistance but also releases proinflammatory mediators into the circulation (48). These pathways converge at the nuclear factor- $\mathrm{\kappa B}(\mathrm{NF}-\kappa \mathrm{B})$ and the c-Jun-amino-terminal kinase (JNK) predisposing to the development of T2DM and promoting hyperglycemia. The common mediators include reactive oxygen species, advanced glycation end-products, C-reactive protein, plasminogen activator inhibitor-1, transforming growth factor- $\beta$ which contribute to not only the development of liver fibrosis and cirrhosis but also other cardiometabolic complications. Therefore, classically, NASH is a proinflammatory state of the body, mediated mostly through transcriptional activation of genes by the NF- $\kappa B$ pathway in the affected hepatocyte. Additionally, it creates a proinflammatory state, which in combination with oxidative stress induced by higher angiotensin II combine to produce epithelial dysfunction in the kidneys. These vascular changes may lead to worsening of CKD triggered by comorbidities of metabolic syndrome $(49,50)$.

\section{Atherogenic dyslipidemia and endothelial dysfunction}

Patients with NASH have atherogenic dyslipidemia which predisposes not only to an increased risk of atherosclerotic vascular disease but also leads to renal endothelial dysfunction. Stimulation of the renin-angiotensin system increases the oxidative stress by concomitant increase in the circulating levels of angiotensin II $(51,52)$. Adiponectin, a protective molecule released from adipose tissues, is decreased in NASH patients. Adiponectin is hypothesized to be down-regulated by another liver generated protein called fetuin-A which is produced in larger quantities in the inflamed liver. With lower levels of adiponectin, a prothrombogenic and proatherogenic environment is generated in the liver and kidneys affecting both the organs. A chronic systemic inflammation and the procoagulant state in NASH contribute to the development and progression of CKD via the effects on renal endothelial cells (53-57).

\section{Intestinal dysbiosis}

Intestinal dysbiosis refers to a perturbed state of the normal intestinal microbiota associated with changes in the composition and number of the gut microbes. Dysbiosis has been reported in context of obesity $(58), \operatorname{NAFLD}(59,60)$ and CKD $(61,62)$. The dysbiosis in the intestine is associated with increased gut permeability, increase in endogenous endotoxemia with an increase in bacterial endotoxin which circulates to the liver. Once in the liver, it promotes progression of NAFLD and enhances an already proinflammatory state existing in the body. While still unproven in humans, new data is available in mice models supporting a state of dysbiosis of intestinal flora in NASH patients. Akkermansia muciniphila and Faecalibacterium prausnitzii are two species of particular interest. High fat diet and genetic obesity have been correlated with a lower level of Akkermansia species in the intestine, which further promotes proinflammatory pathways in the gut (63). On the contrary, in CKD patients, dysbiosis of other gut species such as Bifidobacteriaceae and Lactobacillaceae has been shown. These species influence local production of butyrate and other short chain fatty acids (SCFA). These molecules ordinarily would bind to G-protein coupled receptors (GPCR) present in the colon to maintain intestinal barrier function while down-regulating mucosal inflammation. They also bind to similar receptors present in the hepatocytes to reduce progression of NAFLD to NASH (64). Currently, there are no studies which have particularly evaluated the intestinal dysbiosis in NASH patients with renal dysfunction.

In addition to the above effects on gut barrier function, 
the dysregulated gut bacteriome also influences production of toxic secondary bile acids which may further worsen the mucosal injury. This subtle change in bile acid metabolism has been investigated in the FLINT trial which produced data supporting the use of obeticholic acid for improvement in biopsy proven NASH patients. Obeticholic acid reduces the toxic effect of secondary bile acids such as chenodeoxycholic acid by adding an ethyl group to it, thereby supporting the role of bile acid metabolism in liver injury. Obeticholic acid is associated with decreased intestinal cholesterol transportation and may theoretically reduce atherosclerosis and renal dysfunction, further establishing a link between liver and kidney disease (65).

\section{Challenges in diagnosing CKD in cirrhotics awaiting LT}

Increasingly advanced cirrhotics have or are at increased risk for CKD. Various factors like older age, obesity, presence of diabetes and growing prevalence of NAFLD predispose these patients to the risk of CKD. Further, there is emerging data to suggest a closed intertwined connection between AKI and CKD in cirrhotics (66). The challenge remains the diagnosis of CKD in this extremely sick group of patients. CKD is usually defined as eGFR of less than $60 \mathrm{~mL} / \mathrm{min} / \mathrm{m}^{2}$ for more than 3 months (67). The diagnosis of CKD in advanced cirrhotics is difficult and mostly relies on creatinine-based equations or microscopic urinalysis which does not provide an accurate assessment of the renal functions when compared to the gold standard measures of glomerular filtration rate (GFR) utilising I125iothalamate, iohexol or inulin clearance $(68,69)$. This is because serum creatinine underestimates the degree and severity of renal functions as the levels are influenced by various extraneous factors in patients with advanced cirrhosis. Emerging data has suggested the superiority of Cystatin $\mathrm{C}(\mathrm{Cys} \mathrm{C})$ and equations based on $\mathrm{Cys} C$ to be more accurate as compared to the creatinine-based equations for GFR estimation (67-70). In a recent study, Asrani and colleagues developed a new equation the "GRAIL" equation for determining the renal functions in patients with advanced cirrhotics pre and post-liver transplantation (71). The presence of albuminuria or proteinuria in urine could also be considered as one of the strongest marker of severe renal dysfunction or progression together with the estimation of glomerular filtration for the diagnosis of CKD. Traditionally, filtration of albumin by the glomerulus is followed by receptor-mediated endocytosis by the tubular cells and therefore urine albuminuria reflects the combined process of either glomerular or tubular dysfunction. The integrity of the glomerular filtration barrier depends on the endothelium, the glomerular basement membrane, and the podocytes. However, regardless of the origin of albumin leakage, there is emerging data to suggest that albuminuria itself causes tubulointerstitial damage through activation of proinflammatory mediators which in-turn is associated with progressive decline in renal functions (72). We recently reported an increased prevalence of tubulointerstitial damage and presence of non-diabetic renal disease in histopathological specimens of decompensated cirrhotics with diabetes and abnormal urine microscopy (66). Quantification of the amount and type of albumin in urine as well as tubulointerstitial changes in context of NASH related renal dysfunction needs to be evaluated in future studies. Particularly, the utility of urine microscopy and renal specific biomarkers should be further explored in context of NASH patients with renal dysfunction. This is because, kidney biopsies even though gold standard for diagnosis of CKD are rarely performed in cirrhotics because of the associated risk of complications with ascites and coagulopathy. The approach to renal dysfunction in NAFLD patients has been depicted in Figure 2.

\section{Pharmacotherapy for NAFLD with or without renal dysfunction}

There is currently no definite pharmacotherapy that has been evaluated for renal dysfunction in context of NAFLD patients. Therefore, treatment strategies targeting NAFLD and the associated cardiometabolic factors could also improve renal function (Figure 3). The mainstay of management of NAFLD remains the lifestyle interventions which include dietary, exercise and even bariatric surgery in morbidly obese who fail these or have cardiometabolic risk factors (73-77). Recently, various endoscopic modalities have shown a potential for weight reduction in NASH patients $(78,79)$. Various drugs like pioglitazone $(80)$, obeticholic acid (81), statins (82), metformin $(73,74)$, glucagonlike peptide receptor agonists (83) and anti-oxidants (84) have shown potential in NAFLD. It is quite possible that the same drugs could also improve renal dysfunction by acting on the liver and associated cardiometabolic diseases like diabetes, hypertension and dyslipidemia. This is a speculation which needs to be investigated in randomized controlled trials in context of NAFLD patients with renal 


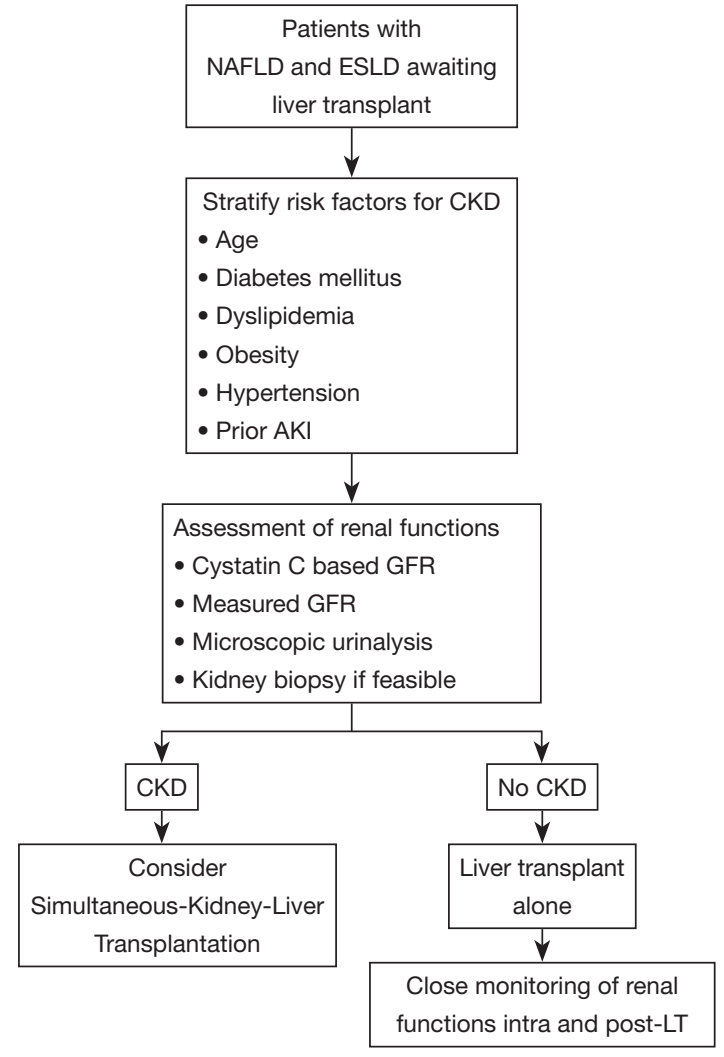

Figure 2 Approach to renal dysfunction in a cirrhotic awaiting liver transplantation with non-alcoholic fatty liver disease. Patients with non-alcoholic liver disease should be stratified based on risk factors i.e., advanced age, obesity, diabetes mellitus, dyslipidemia and hypertension and also those with prior AKI for possible CKD. In patients with risk factors and diagnosis of chronic kidney disease (CKD), the decision to proceed for simultaneous liver-kidney transplant should be done while in the remaining liver transplant alone with close follow up of kidney functions both intra and postoperatively should be done.

dysfunction.

\section{NASH patients as LT candidates}

As LT candidates, NASH patients pose a complex dilemma to the transplant team. As such, no singular stratification scale exists for NASH patients that takes into account all aspects of the disease to assist in prognostication. The multisystemic abnormalities mentioned earlier affect outcome in different ways in each patient, making it imperative to assess each patient differently. Small scale studies have been done to examine the outcome of LT in obese NASH patients. These individuals underwent bariatric surgery with LT, resulting in decreased postoperative weight and improved LT outcomes (85). Studies have indicated poorer LT outcomes, longer hospital stays and higher graft rejection in patients with pretransplant diabetes. Wong and colleagues collected data from the United Network for Organ Sharing and Organ Procurement and Transplantation Network (OPTN) registry from 2004 through 2013 for patients on LT waitlist with different etiologies (86). They observed that compared to patients who had an alcohol, viral or alcoholic/viral etiology, patients with NASH were more obese, more likely to have T2DM and also decreased renal functions. Previous research has shown that patients who have NAFLD have a higher prevalence of CKD than patients who do not have NAFLD (87). Jia et al. in a retrospective study evaluated patients with type 2 diabetes and divided them into two groups based on NAFLD status. The cumulative incidence of CKD and CKD staging was compared between the two groups. They found a higher prevalence of CKD patients in NAFLD as compared to the other group (88).

In a very recent study, Singal and colleagues confirmed that NASH currently is the most common indication for simultaneous liver-kidney (SLK) transplantation considering a significant influence of NASH on impairment in renal functions. They evaluated 38,533 patients from the United Network for Organ Sharing database from 2002 to 2011 for deceased donor LT for patients of liver cirrhosis with different etiologies. They found an increase in the SLK performed for patients with NASH and cryptogenic cirrhosis from $6.3 \%$ in $2002-2003$ to $19.2 \%$ in $2010-2011$. Contrary to this, the trends remained unchanged for patients with other etiology of liver disease. Even though there was no difference in the 5 -year outcomes, however, kidney graft outcome was worse for patients with NASH with higher incidence of kidney graft loss among patients with NASH and cryptogenic cirrhosis when compared to other groups after matching for other characteristics (89). Therefore, studies have demonstrated a higher prevalence of CKD in patients with NASH awaiting LT as compared to other etiologies of liver disease. Furthermore, for patients on the waiting list, the slow progressive nature of the disease often becomes a curse. These patients present with a lower Model for End-Stage Liver Disease (MELD) score and are often placed lower on the waiting list. Patients with ALD had a significantly higher mean score at time of waitlist registration than other registrants. However, after multivariate adjustment, patients with ALD were less 


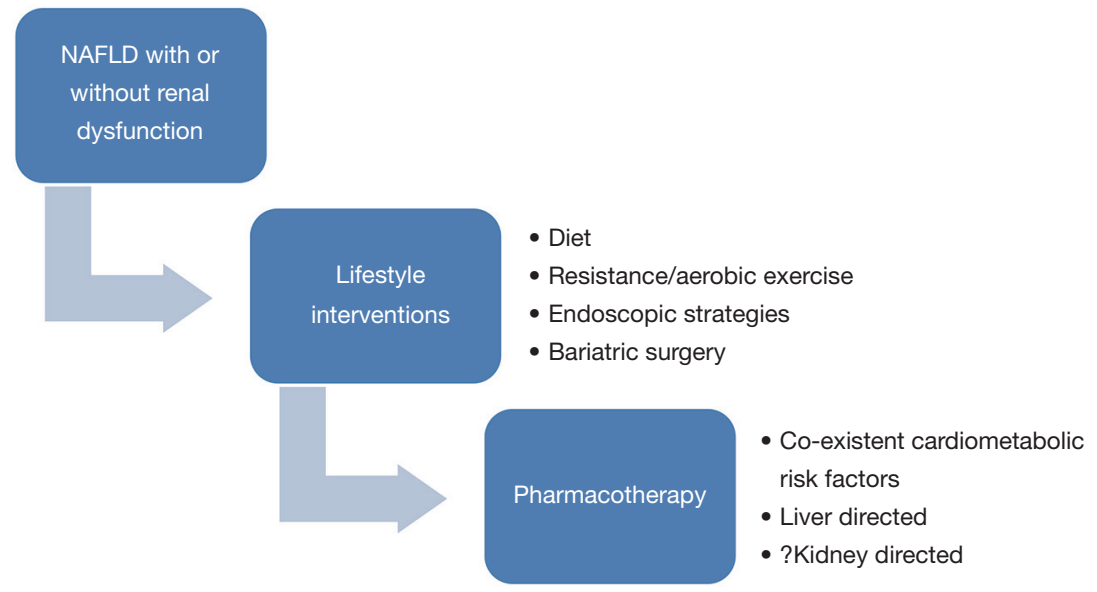

Figure 3 Management of non-alcoholic fatty liver disease with or without associated renal dysfunction.

likely to die within 90 days when compared with patients of NASH $(\mathrm{P}<0.001)$. Similar results were noted by the team for HCV vs. NASH patients (90).

\section{Concerns during transplantation for patients with renal compromise}

During transplant, a number of hemodynamic and electrolyte abnormalities occur as a direct consequence of the steps of transplantation (90). Considering a higher prevalence of renal dysfunction in NASH patients the need of intraoperative hemodialysis could be higher when compared to patients with other etiologies. University of California, Los Angeles did the first study to outline data showing outcomes of intraoperative hemodialysis as compared to continuous renal replacement therapy in dialysis free outcomes post-LT. The team at UCLA did not specifically include NASH as an etiology for their cohort, but renal dysfunction can be extrapolated to be the same if not higher in NASH patients as compared to other etiologies (91). Zimmerman et al. in another study, subsequently showed similar postoperative survival (evaluated at 17.7 months follow-up) in patients who had pre-transplant renal dysfunction and required intraoperative RRT (78\%) as compared to patients who did not have any pre-transplant renal dysfunction and consequently received no intraoperative RRT (88\%) (92). Baek et al. further examined the correlation between intra-operative requirement for RRT with the pre-operative status of the kidneys. This study also did not classify etiologically NASH patients (93). The studies did not emphasize the need of intraoperative renal replacement therapy in patients with pre-transplant chronic renal dysfunction. Over and above, for evaluating the need of RRT requirement intraoperatively, a look at surgical and anesthesia techniques is a must apart from pre-transplant renal functions. Cavapreserving piggyback $(\mathrm{PB})$ technique involves only partial clamping of inferior vena cava during the anhepatic phase of transplantation may prevent hemodynamic instability as compared to the classic cava replacement (CR) $(94,95)$. Amidst conflicting results regarding the use of venovenous bypass (VVB) during caval clamping on the post-LT incidence of acute kidney injury (AKI) and declining use of venovenous bypass in many centres around the world, a study done in China examining 10 years of data brings to light a remarkable finding of reduction in the incidence of AKI in patients in the VVB group when compared to the other group (96).

\section{Post-transplant renal dysfunction}

In the post-transplant period, most recipients are managed with steroid based calcineurin inhibitor (CNI) containing immunosuppressive regimens. In addition to the nephrotoxic side effect of commonly used immunosuppression, there are multiple metabolic effects that interact with existing abnormalities and make postoperative phase difficult for the patient and physician alike. High dose steroid and tacrolimus may worsen existing diabetes or induce new onset diabetes (NOD). Tacrolimus has also been associated with development of hypertension in the postoperative period. On the contrary, cyclosporine has more common 
association with dyslipidemia. An interesting review by Gitto et al. explored the correlation between metabolic syndrome and renal dysfunction post-LT in patients of NASH $(97,98)$. Post-LT diabetes (incidence 10-64\%), dyslipidemia (incidence 45-69\%) and hypertension (incidence $50-100 \%$ ) can add significantly to morbidity and mortality and independently worsen renal function in these patients. As mentioned before, NASH is a hepatic manifestation of metabolic syndrome and with increased risk of development of each of the components of metabolic syndrome in the post-transplant period, recurrent NASH and de-novo NASH is a major possibility. Bhagat et al. reported that $33 \%$ patients transplanted for NASH related liver disease had biopsy proven NASH within a 6-month period post-LT but not one of these patients required a second LT in the 10-year follow-up period (98). While the possibility of development of NASH in the transplanted liver remains for patients undergoing LT for all etiologies, it is significantly higher at 10-year follow-up in those undergoing transplantation for NASH compared to other etiologies (32.9\% vs. 10\%) (99). While large scale cohort studies are lacking, most studies place the risk of NASH development post-LT between $20-40 \%$ with variable incidences being attributable to difference in diagnostic methods in each study. Recent data has surfaced about the utility of ACE inhibitors in preventing development of NASH in postLT period. Orlic et al. showed CKD patients and CKDNAFLD patients with angiotensin-converting enzyme inhibitors (ACE-I) or angiotensin receptor blockers (ARBs) had statistically significant lower degree of liver stiffness in comparison to those without these medications (100). Most transplant centers have a standardized immunosuppression protocol. The choice of immunosuppression regime has a major impact on development of renal dysfunction posttransplant. Further, studies have demonstrated a higher prevalence of renal dysfunction in NASH patients when compared to other etiologies. Houlihan et al. studied patients who underwent deceased donor liver transplant (DDLT) for NASH in a retrospective blinded trial. NASH was diagnosed histologically on liver biopsy. Also included in the study were cases with clinical picture of metabolic syndrome and histologic evidence supportive of cryptogenic liver disease. The cohort was compared with a strictly matched control group of patients who underwent DDLT for indications alternative to NASH in the same period of time at the same centre. In the 1-month period post-LT, the incidence of AKI was similar in both groups and could be attributed to previously identified risk factors such as cardiac dysfunction, type of operation, red blood cell transfusion and intraoperative hypotension. However, kidney function beyond a 3-month period post-LT was more common in population diagnosed with NASH. The difference in the eGFR-values between the NASH and non-NASH group was $8.46 \mathrm{~mL} / \mathrm{min} / 1.73 \mathrm{~m}^{2}$ and remained significant 3 -month after transplant. The difference in renal function persisted at 6,12 and 24 months follow-up. The kidney function did not further deteriorate in the NASH group beyond 3 months possibly due to timely switch from CNI based to mycophenolate mofetil (MMF) based immunosuppressive regimen in response to declining renal function. Notably, while $31.2 \%$ of the patients in the NASH group progressed to a clinically relevant stage of chronic kidney injury (CKI stage IIIb) within 2 years, only $8.3 \%$ of the patients in the control group did ( $\mathrm{P}=0.009)$. Significantly more patients in the NASH group were given the renoprotective immunosuppresive regimen containing MMF during followup. The authors attributed the difference in renal function trends to pre-existing extrahepatic pathophysiological factors leading to the development of CKD and NASH, factors that persist after the surgery as well (7). In the ReSpECT study, lower levels and delayed introduction of tacrolimus were associated with lower incidence of renal dialysis. The authors therefore concluded that daclizumab induction, MMF, corticosteroids and delayed reduced-dose tacrolimus were all less nephrotoxic than therapy with standard-dose tacrolimus and corticosteroids without compromise in efficacy or tolerability (101). In another study, sirolimus was shown to have renoprotective effect when compared to tacrolimusbased regimen $(102,103)$. TruneČka et al. performed the DIAMOND Study with the following groups: prolongedrelease tacrolimus, prolonged-release tacrolimus plus basiliximab and prolonged-release tacrolimus delayed until day 5 plus basiliximab. All patients received MMF plus a bolus of corticosteroid (not followed by maintenance steroids). The incidence of acute cellular rejection (BPAR) and renal outcomes were better in the basiliximab group $(104,105)$. Even though these studies have not been designed for NASH patients, however considering a higher incidence of renal dysfunction, low dose tacrolimus regimens should be considered in patients with NASH beyond 3 months as a strategy to prevent renal compromise post-LT.

\section{Status of SLK in CLD resulting from NASH}

A discussion on LT in renally compromised patients cannot be complete without analysing the current status 
of simultaneous kidney liver transplantation (SLKT) in patients diagnosed with NASH and presenting with a high MELD score. OPTN data reflects survival benefit from SLKT from 1-month to 5 years. The controversy surrounding SLKT is driven by scarcity of organs. Lack of comparative tools to compare the liver disease patient compromised by renal dysfunction with a primary renal disease patient prevent efficient allotment of kidneys. The study by Singal et al. found an increase in the SLK performed for group NASH patients which increased from $6.3 \%$ in 2002 to 2003 to $19.2 \%$ in 2010 to 2011 . They further observed a higher incidence of kidney graft loss among the NASH patients as compared to other etiologies of liver disease (89). Amongst the latest data on the subject is a study done by $\mathrm{Yu}$ et al. published in 2019 examining the OPTN registry from October 1987 to June 2016, the authors identified 7,205 SLK transplants and divided them into groups as per BMI. Twenty-nine percent of patients had NASH in the morbidly obese group versus $16.4 \%$ and $4.7 \%$ in the overweight and normal weight groups, respectively. The 5 -year overall patient survival, kidney and liver graft survival were comparable between the three groups. Multivariate analysis identified donor age, diabetes mellitus, and delayed kidney transplant function as risk factors for poor patient and both liver and kidney graft survival (106). All these studies highlight the need for higher quality data to make a medical decision on the indication of SLK in NASH, with due consideration given to the various metabolic abnormalities often inherent to the disease.

\section{Conclusions}

Pathophysiologically, there is an intricate and complex relationship between the liver and kidneys which share a number of common pathways. Emerging data has suggested a higher prevalence of CKD in NAFLD patients, which is governed by the presence and severity of NAFLD independent of concurrent metabolic factors. The challenge remains the diagnosis of CKD which relies in majority on GFR-estimating equations and urine microscopy. Biomarkers like $\mathrm{Cys} C$ remain attractive strategy to diagnose and screen renal dysfunction and presence of underlying CKD in NAFLD patients. The diagnosis of significant renal dysfunction pre-transplant has an implication on intraoperative surgical strategy and need of renal replacement therapy for preservation of kidney functions post-LT. Further, the choice immunosuppression is also governed by the status of the kidney's pre-transplant with use of reno-protective immunosuppression regimens. Screening for presence and severity of renal dysfunction is also pertinent in stratifying the need for SLKT as against LT alone in these patients. Till date, in the absence of treatment options for NAFLD, lifestyle modifications and aggressive management of cardiometabolic factors could be helpful in decreasing the incidence of CKD. Prospective controlled trials focusing on NAFLD and development of CKD are needed to definitively assess the existence of a complex and a possible intertwined relationship between the two entities.

\section{Acknowledgments}

None.

\section{Footnote}

Conflicts of Interest: The authors have no conflicts of interest to declare.

Ethical Statement: The authors are accountable for all aspects of the work in ensuring that questions related to the accuracy or integrity of any part of the work are appropriately investigated and resolved.

\section{References}

1. Vernon G, Baranova A, Younossi ZM. Systematic review: the epidemiology and natural history of non-alcoholic fatty liver disease and non-alcoholic steatohepatitis in adults. Aliment Pharmacol Ther 2011;34:274-85.

2. Singh S, Kuftinec GN, Sarkar S. Non-alcoholic Fatty Liver Disease in South Asians: A Review of the Literature. J Clin Transl Hepatol 2017;5:76-81.

3. Younossi ZM, Golabi P, de Avila L, et al. The Global Epidemiology of NAFLD and NASH in Patients with type 2 diabetes: A Systematic Review and Meta-analysis. J Hepatol 2019;71:793-801.

4. Kutlu O, Kaleli HN, Ozer E. Molecular Pathogenesis of Nonalcoholic Steatohepatitis- (NASH-) Related Hepatocellular Carcinoma. Can J Gastroenterol Hepatol 2018;2018:8543763.

5. Guzman G, Brunt EM, Petrovic LM, et al. Does nonalcoholic fatty liver disease predispose patients to hepatocellular carcinoma in the absence of cirrhosis? Arch Pathol Lab Med 2008;132:1761-6. 
6. Khukhlina OS, Antoniv AA, Mandryk OY, et al. The role of endothelial dysfunction in the progression mechanisms of non-alcoholic steatohepatitis in patients with obesity and chronic kidney disease. Wiad Lek 2019;72:523-6.

7. Houlihan DD, Armstrong MJ, Davidov Y, et al. Renal function in patients undergoing transplantation for nonalcoholic steatohepatitis cirrhosis: time to reconsider immunosuppression regimens? Liver Transpl 2011;17:1292-8.

8. Targher G, Byrne CD. Non-alcoholic fatty liver disease: an emerging driving force in chronic kidney disease. Nat Rev Nephrol 2017;13:297-310.

9. Pacifico L, Bonci E, Andreoli GM, et al. The Impact of Nonalcoholic Fatty Liver Disease on Renal Function in Children with Overweight/Obesity. Int J Mol Sci 2016;17:8.

10. Musso G, Gambino R, Tabibian JH, et al. Association of non-alcoholic fatty liver disease with chronic kidney disease: a systematic review and meta-analysis. PLoS Med 2014;11:e1001680.

11. Mantovani A, Zaza G, Byrne CD, et al. Nonalcoholic fatty liver disease increases risk of incident chronic kidney disease: A systematic review and meta-analysis. Metabolism 2018;79:64-76.

12. Fan JG, Kim SU, Wong VW. New trends on obesity and NAFLD in Asia. J Hepatol 2017;67:862-73.

13. Lazo M, Clark JM. The epidemiology of nonalcoholic fatty liver disease: A global perspective. Semin Liver Dis 2008;28:339-50.

14. de Vries AP, Ruggenenti P, Ruan XZ, et al. Fatty kidney: emerging role of ectopic lipid in obesity-related renal disease. Lancet Diabetes Endocrinol 2014;2:417-26.

15. Salvatore SP, Chevalier JM, Kuo SF, et al. Kidney disease in patients with obesity: It is not always obesityrelated glomerulopathy alone. Obes Res Clin Pract 2017;11:597-606.

16. Hsu CY, McCulloch CE, Iribarren C, et al. Body mass index and risk for end-stage renal disease. Ann Intern Med 2006;144:21-8.

17. Munkhaugen J, Lydersen S, Widerøe TE, et al. Prehypertension, obesity, and risk of kidney disease: 20 year follow-up of the HUNT I study in Norway. Am J Kidney Dis 2009;54:638-46.

18. Kurella M. Metabolic syndrome and the risk for chronic kidney disease among nondiabetic adults. J Am Soc Nephrol 2005;16:2134-40.

19. Chagnac A, Zingerman B, Rozen-Zvi B, et al. Consequences of Glomerular Hyperfiltration: The Role of Physical Forces in the Pathogenesis of Chronic Kidney Disease in Diabetes and Obesity. Nephron 2019;143:38-42.

20. Oterdoom LH, de Vries APJ, Gansevoort RT, et al. Fasting insulin modifies the relation between age and renal function. Nephrol Dial Transplant 2007;22:1587-92.

21. Tomaszewski M, Charchar FJ, Maric C, et al. Glomerular hyperfiltration: a new marker of metabolic risk. Kidney Int 2007;71:816-21.

22. Serra A, Romero R, Lopez D, et al. Renal injury in the extremely obese patients with normal renal function. Kidney Int 2008;73:947-55.

23. Navaneethan SD, Yehnert H, Moustarah F, et al. Weight loss interventions in chronic kidney disease: a systematic review and meta-analysis. Clin J Am Soc Nephrol 2009;4:1565-74.

24. Björntorp P. "Portal" adipose tissue as a generator of risk factors for cardiovascular disease and diabetes. Arteriosclerosis 1990;10:493-96.

25. Steinberg D, Parthasarathy S, Carew TE, et al. Beyond cholesterol. Modifications of low-density lipoprotein that increase its atherogenicity. N Engl J Med 1989;320:915-24.

26. Armstrong MJ, Adams LA, Canbay A, et al. Extrahepatic complications of nonalcoholic fatty liver disease. Hepatology 2014;59:1174-97.

27. Rickards E. Remarks on the fatty transformation of the kidney. BMJ 1883;2:2-3.

28. Chen HM, Liu ZH, Zeng CH, et al. Podocyte lesions in patients with obesity-related glomerulopathy. Am J Kidney Dis 2006;48:772-9.

29. Ananthakrishnan S, Kaysen GA. Treatment of Hyperlipidemia Changes With Level of Kidney FunctionRationale. Adv Chronic Kidney Dis 2016;23:247-54.

30. Rahman M, Yang W, Akkina S, et al. Relation of serum lipids and lipoproteins with progression of CKD: The CRIC study. Clin J Am Soc Nephrol 2014;9:1190-8.

31. Adams LA, Anstee QM, Tilg H, et al. Non-alcoholic fatty liver disease and its relationship with cardiovascular disease and other extrahepatic diseases. Gut 2017;66:1138-53.

32. Targher G, Marchesini G, Byrne CD, et al. Risk of type 2 diabetes in patients with non-alcoholic fatty liver disease: Causal association or epiphenomenon? Diabetes Metab 2016;42:142-56.

33. Loomba R, Abraham M, Unalp A, et al. Nonalcoholic Steatohepatitis Clinical Research Network. Association between diabetes, family history of diabetes, and risk of nonalcoholic steatohepatitis and fibrosis. Hepatology 2012;56:943-51.

34. Williams CD, Stengel J, Asike MI, et al. Prevalence 
of nonalcoholic fatty liver disease and nonalcoholic steatohepatitis among a largely middle-aged population utilizing ultrasound and liver biopsy: A prospective study. Gastroenterology 2011;140:124-31.

35. Musso G, Gambino R, Cassader M, et al. Meta-analysis: Natural history of non-alcoholic fatty liver disease (NAFLD) and diagnostic accuracy of non-invasive tests for liver disease severity. Ann Med 2011;43:617-49.

36. Lonardo A, Bellentani S, Argo CK, et al. Epidemiological modifiers of non-alcoholic fatty liver disease: Focus on high-risk groups. Dig Liver Dis 2015;47:997-1006.

37. Machado MV, Cortez-Pinto H. Non-invasive diagnosis of non-alcoholic fatty liver disease. A critical appraisal. J Hepatol 2013;58:1007-19.

38. Ballestri S, Zona S, Targher G, et al. Nonalcoholic fatty liver disease is associated with an almost twofold increased risk of incident type 2 diabetes and metabolic syndrome. Evidence from a systematic review and meta-analysis. J Gastroenterol Hepatol 2016;31:936-44.

39. Yamada T, Fukatsu M, Suzuki S, et al. Fatty liver predicts impaired fasting glucose and type 2 diabetes mellitus in Japanese undergoing a health checkup. J Gastroenterol Hepatol 2010;25:352-6.

40. Bae JC, Cho YK, Lee WY, et al. Impact of nonalcoholic fatty liver disease on insulin resistance in relation to HbA1c levels in nondiabetic subjects. Am J Gastroenterol 2010;105:2389-95.

41. McPherson S, Hardy T, Henderson E, et al. Evidence of NAFLD progression from steatosis to fibrosingsteatohepatitis using paired biopsies: Implications for prognosis and clinical management. J Hepatol 2015;62:1148-55.

42. Hossain N, Afendy A, Stepanova M, et al. Independent predictors of fibrosis in patients with nonalcoholic fatty liver disease. Clin Gastroenterol Hepatol 2009;7:1224-9.

43. Fracanzani AL, Valenti L, Bugianesi E, et al. Risk of severe liver disease in nonalcoholic fatty liver disease withnormal aminotransferase levels: A role for insulin resistance and diabetes. Hepatology 2008;48:792-8.

44. Targher G, Chonchol M, Bertolini L, et al. Increased risk of CKD among type 2 diabetics with nonalcoholic fatty liver disease. J Am Soc Nephrol 2008;19:1564-70.

45. Targher G, Mantovani A, Pichiri I, et al. Nonalcoholic fatty liver disease is independently associated with an increased incidence of chronic kidney disease in patients with type 1 diabetes. Diabetes Care 2014;37:1729-36.

46. Nampoothiri RV, Duseja A, Rathi M, et al. Renal Dysfunction in Patients With Nonalcoholic Fatty Liver
Disease is Related to the Presence of Diabetes Mellitus and Severity of Liver Disease. J Clin Exp Hepatol 2019;9:22-8.

47. Scorletti E, Calder PC, Byrne CD. Non-alcoholic fatty liver disease and cardiovascular risk: metabolic aspects and novel treatments. Endocrine 2011;40:332-43.

48. Lim S, Meigs JB. Links between ectopic fat and vascular disease in humans. Arterioscler Thromb Vasc Biol 2014;34:1820-6.

49. Sharma M, Mitnala S, Vishnubhotla RK, et al. The Riddle of Nonalcoholic Fatty Liver Disease: Progression From Nonalcoholic Fatty Liver to Nonalcoholic Steatohepatitis. J Clin Exp Hepatol 2015;5:147-58.

50. Willy JA, Young SK, Stevens JL, et al. CHOP links endoplasmic reticulum stress to NF-kappaB activation in the pathogenesis of nonalcoholic steatohepatitis. Mol Biol Cell 2015;26:2190-204.

51. Kamide K. Role of renin-angiotensin-aldosterone system in metabolic syndrome and obesity-related hypertension. Curr Hypertens Rev 2014;9:238-45.

52. Cabandugama PK, Gardner MJ, Sowers JR. The renin angiotensin aldosterone system in obesity and hypertension: roles in the cardiorenal metabolic syndrome. Med Clin North Am 2017;101:129-37.

53. Kronenberg, F. Emerging risk factors and markers of chronic kidney disease progression. Nat Rev Nephrol 2009;5:677-89.

54. Massy ZA, Stenvinkel P, Drueke TB. The role of oxidative stress in chronic kidney disease. Semin Dial 2009;22:405-8.

55. Vlassara H. Role of oxidants/inflammation in declining renal function in chronic kidney disease and normal aging. Kidney Int Suppl 2009;114:S3-11.

56. Bugianesi E, Pagotto U, Manini R, et al. Plasma adiponectin in nonalcoholic fatty liver is related to hepatic insulin resistance and hepatic fat content, not to liver disease severity. J Clin Endocrinol Metab 2005;90:3498-504.

57. Targher G, Bertolini L, Rodella S, et al. Associations between plasma adiponectin concentrations and liver histology in patients with nonalcoholic fatty liver disease. Clin Endocrinol (Oxf) 2006;64:679-83.

58. Mehal WZ. The Gordian Knot of dysbiosis, obesity and NAFLD. Nat Rev Gastroenterol Hepatol 2013;10:637-44.

59. Wieland A, Frank DN, Harnke B, et al. Systematic review: microbial dysbiosis and nonalcoholic fatty liver disease. Aliment Pharmacol Ther 2015;42:1051-63.

60. Boursier J. The severity of nonalcoholic fatty liver disease is associated with gut dysbiosis and shift in the metabolic function of the gut microbiota. Hepatology 2016;63:764-75.

61. Sampaio-Maia B, Simoes-Silva L, Pestana M, et al. The 
role of the gut microbiome on chronic kidney disease. Adv Appl Microbiol 2016;96:65-94.

62. Nallu A, Sharma S, Ramezani A, et al. Gut microbiome in chronic kidney disease: challenges and opportunities. Transl Res 2017;179:24-37.

63. Schneeberger M, Everard A, Gomez-Valades AG, et al. Akkermansia muciniphila inversely correlates with the onset of inflammation, altered adipose tissue metabolism and metabolic disorders during obesity in mice. Sci Rep 2015;5:16643.

64. Bashiardes S, Shapiro H, Rozin S, et al. Non-alcoholic fatty liver and the gut microbiota. Mol Metab 2016;5:782-94.

65. Neuschwander-Tetri BA, Loomba R, Sanyal AJ, et al. Farnesoid X nuclear receptor ligand obeticholic acid for non-cirrhotic, non-alcoholic steatohepatitis (FLINT): a multicentre, randomised, placebo-controlled trial. Lancet 2015;385:956-65.

66. Maiwall R, Rao Pasupuleti SS, Bihari C, et al. Incidence, Risk Factors, and Outcomes of Transition of Acute Kidney Injury to Chronic Kidney Disease in Cirrhosis: A Prospective Cohort Study. Hepatology 2019. [Epub ahead of print].

67. Chawla LS, Bellomo R, Bihorac A, et al. Acute kidney disease and renal recovery: consensus report of the Acute Disease Quality Initiative (ADQI) 16 Workgroup. Nat Rev Nephrol 2017;13:241-57.

68. Allen AM, Kim WR, Larson JJ, et al. Serum Cystatin C as an indicator of renal function and mortality in liver transplant recipients. Transplantation 2015;99:1431-5.

69. Mindikoglu AL, Dowling TC, Weir MR, et al. Performance of chronic kidney disease epidemiology collaboration creatinine-cystatin $\mathrm{C}$ equation for estimating kidney function in cirrhosis. Hepatology 2014;59:1532-42.

70. Maiwall R, Sarin SK, Moreau R. Acute kidney injury in acute on chronic liver failure. Hepatol Int 2016;10:245-57.

71. Asrani SK, Jennings LW, Trotter JF, et al. A Model for Glomerular Filtration Rate Assessment in Liver Disease (GRAIL) in the Presence of Renal Dysfunction. Hepatology 2019;69:1219-30.

72. Lambers Heerspink HJ, Gansevoort RT. Albuminuria Is an Appropriate Therapeutic Target in Patients with CKD: The Pro View. Clin J Am Soc Nephrol 2015;10:1079-88

73. Lassailly G, Caiazzo R, Pattou F, et al. Perspectives on treatment for non-alcoholic steatohepatitis. Gastroenterology 2016;150:1835-48.

74. Corey KE, Rinella ME. Medical and surgical treatment options for nonalcoholic steatohepatitis. Dig Dis Sci 2016;61:1387-97.
75. Rotman Y, Sanyal AJ. Current and upcoming pharmacotherapy for non-alcoholic fatty liver disease. Gut 2017;66:180-90.

76. Zhang $\mathrm{H}$, Zhang X, Hu C, et al. Exenatide reduces urinary transforming growth factor-beta1 and type IV collagen excretion in patients with type 2 diabetes and microalbuminuria. Kidney Blood Press Res 2012;35:483-8.

77. Vilar-Gomez E. Improvement in liver histology due to lifestyle modification is independently associated with improved kidney function in patients with non-alcoholic steatohepatitis. Aliment Pharmacol Ther 2017;45:332-44.

78. Vyas D, Deshpande K, Pandya Y. Advances in endoscopic balloon therapy for weight loss and its limitations. World J Gastroenterol 2017;23:7813-7.

79. Kumar N. Endoscopic therapy for weight loss: Gastroplasty, duodenal sleeves, intragastric balloons, and aspiration. World J Gastrointest Endosc 2015;7:847-59.

80. Cusi K. Long-term pioglitazone treatment for patients with nonalcoholic steatohepatitis and prediabetes or type 2 diabetes mellitus: a randomized trial. Ann Intern Med 2016;165:305-15.

81. Neuschwander-Tetri BA. Farnesoid X nuclear receptor ligand obeticholic acid for non-cirrhotic, nonalcoholic steatohepatitis (FLINT): a multicentre, randomised, placebo-controlled trial. Lancet 2015;385:956-65.

82. Palmer SC. HMG CoA reductase inhibitors (statins) for people with chronic kidney disease not requiring dialysis. Cochrane Database Syst Rev 2014;5:CD007784.

83. Seghieri M, Christensen AS, Andersen A, et al. Front Endocrinol (Lausanne) 2018;9:649.

84. Sanyal AJ. Pioglitazone, vitamin E, or placebo for nonalcoholic steatohepatitis. N Engl J Med 2010;362:1675-85.

85. Heimbach JK, Watt KD, Poterucha JJ, et al. Combined liver transplantation and gastric sleeve resection for patients with medically complicated obesity and end-stage liver disease. Am J Transplant 2013;13:363-8.

86. Wong RJ, Aguilar M, Cheung R, et al. Nonalcoholic steatohepatitis is the second leading etiology of liver disease among adults awaiting liver transplantation in the United States. Gastroenterology 2015;148:547-55.

87. Mikolasevic I, Milic S, Turk Wensveen T, et al. Nonalcoholic fatty liver disease - A multisystem disease? World J Gastroenterol 2016;22:9488-505.

88. Jia G, Di F, Wang Q, Shao J, et al. Non-Alcoholic Fatty Liver Disease Is a Risk Factor for the Development of Diabetic Nephropathy in Patients with Type 2 Diabetes Mellitus. PLoS One 2015;10:e0142808. 
89. Singal AK, Hasanin M, Kaif $M$, et al. Nonalcoholic Steatohepatitis is the Most Rapidly Growing Indication for Simultaneous Liver Kidney Transplantation in the United States. Transplantation 2016;100:607-12.

90. Vitin A, Muczynski K, Bakthavatsalam R, et al. Treatment of severe lactic acidosis during the pre-anhepatic stage of liver transplant surgery with intraoperative hemodialysis. J Clin Anesth 2010;22:466-72.

91. Nadim MK, Annanthapanyasut W, Matsuoka L, et al. Intraoperative hemodialysis during liver transplantation: a decade of experience. Liver Transpl 2014;20:756-64.

92. Zimmerman MA, Selim M, Kim J, et al. Outcome analysis of continuous intraoperative renal replacement therapy in the highest acuity liver transplant recipients: A singlecenter experience. Surgery 2017;161:1279-86.

93. Baek SD, Jang M, Kim W, et al. Benefits of Intraoperative Continuous Renal Replacement Therapy During Liver Transplantation in Patients With Renal Dysfunction. Transplant Proc 2017;49:1344-50.

94. Cabezuelo JB, Ramirez P, Acosta F, et al. Does the standard vs. piggyback surgical technique affect the development of early acute renal failure after orthotopic liver transplantation? Transplant Proc 2003;35:1913-4.

95. Barbas AS, Levy J, Mulvihill MS, et al. Liver Transplantation Without Venovenous Bypass: Does Surgical Approach Matter? Transplant Direct 2018;4:e348.

96. Sun K, Hong F, Wang Y, et al. Venovenous Bypass Is Associated With a Lower Incidence of Acute Kidney Injury After Liver Transplantation in Patients With Compromised Pretransplant Renal Function. Anesth Analg 2017;125:1463-70.

97. Gitto S, Villa E. Non-Alcoholic Fatty Liver Disease and Metabolic Syndrome after Liver Transplant. Int J Mol Sci 2016;17:490.

98. Mikolasevic I, Orlic L, Hrstic I, et al. Metabolic syndrome and non-alcoholic fatty liver disease after liver or kidney transplantation. Hepatol Res 2016;46:841-52.

doi: $10.21037 / \operatorname{tgh} .2019 .10 .11$

Cite this article as: Maiwall R, Gupta M. Peri-transplant renal dysfunction in patients with non-alcoholic steatohepatitis undergoing liver transplantation. Transl Gastroenterol Hepatol 2020;5:18.
99. Yalamanchili K, Saadeh S, Klintmalm GB, et al. Nonalcoholic fatty liver disease after liver transplantation for cryptogenic cirrhosis or nonalcoholic fatty liver disease. Liver Transpl 2010;16:431-9.

100. Orlic L, Mikolasevic I, Lukenda V, et al. Nonalcoholic fatty liver disease and the renin-angiotensin system blockers in the patients with chronic kidney disease. Wien Klin Wochenschr 2015;127:355-62.

101. Neuberger JM, Mamelok RD, Neuhaus P, et al. Delayed introduction of reduced-dose tacrolimus, and renal function in liver transplantation: the 'ReSpECT' study. Am J Transplant 2009;9:327-36.

102. Duhart BT Jr, Ally WA, Krauss AG, et al. The benefit of sirolimus maintenance immunosuppression and rabbit antithymocyte globulin induction in liver transplant recipients that develop acute kidney injury in the early postoperative period. J Transplant 2015;2015:926168.

103. Schnitzbauer AA, Sothmann J, Baier L, et al. Calcineurin Inhibitor Free De Novo Immunosuppression in Liver Transplant Recipients With Pretransplant Renal Impairment: Results of a Pilot Study (PATRON07). Transplantation 2015;99:2565-75.

104. Trune ka P, Klempnauer J, Bechstein WO, et al. Renal Function in De Novo Liver Transplant Recipients Receiving Different Prolonged-Release Tacrolimus Regimens-The DIAMOND Study. Am J Transplant 2015;15:1843-54.

105. Dopazo C, Charco R, Caralt M, et al. Low Total Dose of Anti-Human T-Lymphocyte Globulin (ATG) Guarantees a Good Glomerular Filtration Rate after Liver Transplant in Recipients with Pretransplant Renal Dysfunction. Can J Gastroenterol Hepatol 2018;2018:1672621.

106. Yu JW, Gupta G, Kang L, et al. Obesity does not significantly impact outcomes following simultaneous liver kidney transplantation: review of the UNOS database - a retrospective study. Transpl Int 2019;32:206-17. 